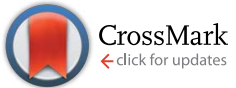

Cite this: RSC Adv., 2015, 5, 2383

Received 5th September 2014 Accepted 26th November 2014

DOI: $10.1039 / c 4 r a 09839 k$

www.rsc.org/advances

\section{Attapulgite-doped electrospun poly(lactic-co- glycolic acid) nanofibers enable enhanced osteogenic differentiation of human mesenchymal stem cells $\dagger$}

\author{
Zhe Wang, ${ }^{a}$ Yili Zhao, ${ }^{b}$ Yu Luo, ${ }^{a}$ Shige Wang, ${ }^{c}$ Mingwu Shen, ${ }^{a}$ Helena Tomás, ${ }^{d}$ \\ Meifang Zhuc and Xiangyang Shi ${ }^{\star a c d}$
}

\begin{abstract}
The extracellular matrix mimicking property of electrospun polymer nanofibers affords their uses as an ideal scaffold material for differentiation of human mesenchymal stem cells (hMSCs), which is important for various tissue engineering applications. Here, we report the fabrication of electrospun poly(lactic-coglycolic acid) (PLGA) nanofibers incorporated with attapulgite (ATT) nanorods, a clay material for osteogenic differentiation of hMSCs. We show that the incorporation of ATT nanorods does not significantly change the uniform morphology and the hemocompatibility of the PLGA nanofibers; instead the surface hydrophilicity and cytocompatibility of the hybrid nanofibers are slightly improved after doping with ATT. Alkaline phosphatase activity, osteocalcin secretion, calcium content, and von Kossa staining assays reveal that hMSCs are able to be differentiated to form osteoblast-like cells onto both PLGA and PLGA-ATT composite nanofibers in osteogenic medium. Most strikingly, the doped ATT within the PLGA nanofibers is able to induce the osteoblastic differentiation of hMSCs in growth medium without the inducing factor of dexamethasone. The fabricated organic/inorganic hybrid ATT-doped PLGA nanofibers may find many applications in the field of tissue engineering and regenerative medicine.
\end{abstract}

\section{Introduction}

Electrospinning is a simple and versatile nanofabrication technique that can be used to manufacture ultralong nanofibers with controllable diameters ranging from a few nanometers to several micrometers. ${ }^{1,2}$ A wide variety of polymers such as polycaprolactone, ${ }^{3,4}$ polyvinyl pyrrolidone, ${ }^{5,6}$ polyurethane, ${ }^{7}$ polyvinyl acetate, ${ }^{8}$ and poly(lactic-co-glycolic acid) (PLGA) ${ }^{9}$ can be electrospun to form nanofibers. The formed electrospun nanofibers have been applied in various applications, including but not limited to sensors, ${ }^{10,11}$ supercapacitors, ${ }^{12,13}$ dyesensitized solar cells (DSSCs) ${ }^{\mathbf{1 4}, 15}$ wound dressing, ${ }^{\mathbf{1 6}}$ drug delivery, ${ }^{17-21}$ and tissue engineering. ${ }^{22,23}$

\footnotetext{
${ }^{a}$ College of Chemistry, Chemical Engineering and Biotechnology, Donghua University, Shanghai 201620, People's Republic of China. E-mail: xshi@dhu.edu.cn; Fax: +86-2167792306-804; Tel: +86-21-67792656

${ }^{b}$ College of Textiles, Donghua University, Shanghai 201620, People's Republic of China ${ }^{c}$ State Key Laboratory for Modification of Chemical Fibers and Polymer Materials, College of Materials Science and Engineering, Donghua University, Shanghai 201620, People's Republic of China

${ }^{d}$ CQM-Centro de Química da Madeira, Universidade da Madeira, Campus da Penteada, 9000-390 Funchal, Portugal

$\dagger$ Electronic supplementary information (ESI) available: Additional experimental results. See DOI: 10.1039/c4ra09839k
}

To generate nanofibers with desirable mechanical durability and functionality, it is essential to incorporate inorganic components within polymer nanofibers. ${ }^{\mathbf{1 7 2 4 - 2 8}}$ For instance, halloysite nanotubes, ${ }^{17,29,30}$ nanohydroxyapatite, ${ }^{26,27}$ and laponite $^{25,31}$ have been incorporated within PLGA nanofibers for drug delivery and tissue engineering applications. In particular, it has been shown that the incorporation of these inorganic components is able to not only improve the mechanical durability of the nanofibers, but also facilitate the attachment and proliferation of cells..$^{25,27,30}$

In our previous work, we have shown that laponite-doped electrospun PLGA nanofibers with extracellular matrix (ECM) mimicking property are able to induce osteogenic differentiation of human mesenchymal stem cells (hMSCs) in the absence of the inducing factor of dexamethasone (DEX). ${ }^{25}$ The osteogenic differentiation of hMSCs is believed to be attributed to the presence of laponite that is a biodegradable nanodisk-shaped layered synthetic aluminosilicate clay material. ${ }^{32}$ It is generally known that a bioceramic such as a calcium magnesium silicate (akermanite) having silicon and/or magnesium elements is able to promote the osteogenic differentiation of MSCs. ${ }^{33}$ Therefore, it is reasonable to hypothesize that polymer nanofibers incorporated with other clay materials containing silicon and/or magnesium elements may also be able to promote the osteogenic differentiation of MSCs. 
Attapulgite (ATT) is a naturally occurring clay material, a kind of magnesium aluminium phyllosilicate with formula (Mg,Al $)_{2} \mathrm{Si}_{4} \mathrm{O}_{10}(\mathrm{OH}) \cdot 4\left(\mathrm{H}_{2} \mathrm{O}\right)$. It has been reported that ATT/ polymer composite materials display improved mechanical durability due to the presence of ATT. ${ }^{34-37}$ For instance, Tian et al. prepared ATT-doped nylon 6 electrospun nanofibers and showed that in the presence of a small percentage (1\% and $2 \%$ ) of ATT, the nanofibers displayed substantially improved mechanical durability. ${ }^{36}$

In this present study, we prepared electrospun ATT-doped PLGA nanofibers for osteogenic differentiation of hMSCs. The formed ATT-incorporated electrospun PLGA hybrid nanofibers were characterized via different techniques. Scanning electron microscopy (SEM), tensile tests, and water contact angle measurements were used to investigate the influence of the incorporated ATT on the morphology, mechanical properties, and surface hydrophilicity of the hybrid fibers, respectively. The cytocompatibility of the hybrid nanofibers was evaluated by cell viability assay and SEM morphology observation of hMSCs cultured onto the prepared fibrous scaffolds. The hemocompatibility of the ATT-doped PLGA nanofibers was evaluated via hemolytic and anticoagulant assays. Finally, the osteogenic differentiation of hMSCs cultured onto ATT-doped PLGA nanofibers was quantitatively investigated by measuring the alkaline phosphatase (ALP) activity, osteocalcin secretion, and cellular calcium content, and qualitatively evaluated by von Kossa staining of calcium phosphate crystals produced in culture. To our knowledge, this is the first report related to the use of ATT-doped PLGA nanofibers as a scaffolding material for osteogenic differentiation of stem cells.

\section{Experimental}

\section{Materials}

$\operatorname{PLGA}\left(M_{\mathrm{w}}=81000 \mathrm{~g} \mathrm{~mol}^{-1}\right)$ with a lactic acid/glycolic acid ratio of 50 : 50 was purchased from Jinan Daigang Biotechnology Co., Ltd. (Jinan, China). ATT was from Mingguang Jianxi Dongfeng Mine Products Factory (Mingguang, China). Dimethylsulfoxide (DMSO), tetrahydrofuran (THF), $N, N$-dimethyl formamide (DMF), silver nitrate, and sodium thiosulfate were from Sinopharm Chemical Reagent Co., Ltd. (Shanghai, China). hMSCs derived from umbilical cord blood and heparin stabilized human blood were kindly provided by Shanghai First People's Hospital (Shanghai, China) and approved by the Ethical Committee of Shanghai First People's Hospital. Dulbecco's Modified Eagle's Medium (DMEM), fetal bovine serum (FBS), phosphate buffered saline (PBS), penicillin, and streptomycin were purchased from Gibco (Carlsbad, CA). 3-(4,5Dimethylthiazol-2-yl)-2,5-diphenyltetrazolium bromide (MTT) was from Shanghai Sangon Biological Engineering Technology \& Services Co., Ltd (Shanghai, China). DEX, betaglycerophosphate (beta-GP), ascorbic acid, resazurin, $p$-nitrophenyl phosphate, and $p$-nitrophenol standard were from Sigma-Aldrich (St Louis, MO). $\mathrm{CaCl}_{2}, \mathrm{NaOH}, \mathrm{MgCl}_{2}$ and formaldehyde were from Merck (Whitehouse Station, NJ). Reporter lysis buffer was from Promega (Madison, WI). Intact human osteocalcin EIA kit was from Biomedical Technologies Inc.
(Boston, MA). BCA Protein Assay Kit was purchased from Shanghai Qianchen Biotechnology Company (Shanghai, China). QuantiChrom Calcium Assay Kit was purchased from Bioassay Systems (Hayward, CA). Water used in all experiments was purified using a Milli-Q Plus 185 water purification system (Millipore, Bedford, MA) with a resistivity higher than $18 \mathrm{M} \Omega \mathrm{cm}$.

\section{Preparation of electrospun ATT-doped PLGA nanofibers}

Following procedures described in our previous study, ${ }^{25-27,31}$ PLGA was dissolved in a mixed solvent of THF-DMF (v/v = $3: 1)$ under magnetic stirring overnight to obtain a homogeneous solution with a concentration of $25 \%(\mathrm{w} / \mathrm{v})$. After that, ATT (1, 2, or $3 \mathrm{wt} \%$ relative to PLGA) was separately added into the above PLGA solution, followed by continuous stirring for $1 \mathrm{~h}$ to obtain a homogeneous solution mixed with different amounts of ATT.

The electrospinning system was set up with a syringe pump with a $10 \mathrm{~mL}$ syringe, a silicone hose, a stainless steel needle with an inner diameter of $0.8 \mathrm{~mm}$, a high voltage power supply, and a thin aluminum foil acting as a collector which was positioned horizontally and grounded. A clamp was used to connect the high voltage power supply with the needle. At a fixed electrical potential of $20 \mathrm{kV}$, steel capillary was charged. The distance between the tip and the collector was set at $15 \mathrm{~cm}$. The electrospinning solution was fed at a speed of $0.8 \mathrm{~mL} \mathrm{~h}^{-1}$ by the syringe pump, and the electrospinning process was carried out under ambient conditions. After electrospinning, aluminum foil covered with the formed nanofibrous mat was taken off from the collector immediately and vacuum dried for at least 48 $\mathrm{h}$ to remove the residual organic solvent and moisture. Finally, the formed nanofibers were removed carefully from the aluminum foil and stored in a desiccator before use. Under these conditions, electrospun PLGA, PLGA-1\%ATT, PLGA-2\% ATT, and PLGA-3\%ATT nanofibers were obtained.

\section{Characterization techniques}

The morphology of ATT and ATT-doped PLGA composite nanofibers was observed using a Hitachi $\mathrm{H}-800$ transmission electron microscope (TEM, Tokyo, Japan) with an accelerating voltage of $200 \mathrm{kV}$. The suspension of ATT $\left(1 \mathrm{mg} \mathrm{mL}^{-1}\right)$ dispersed in THF-DMF was dropped onto a carbon-coated copper grid and air dried before TEM imaging. For the hybrid nanofiber samples, the ATT-doped PLGA nanofibers were directly electrospun onto the carbon-coated copper grid and vacuum dried before TEM imaging. The morphology of the nanofibers was also observed using SEM (JEOL JSM-5600LV, Tokyo, Japan). Before SEM imaging, the samples were sputter-coated with a thin gold film with a thickness of $10 \mathrm{~nm}$. The diameters of the nanofibers were analyzed using the Image $1.40 \mathrm{G}$ software (http://rsb.info.nih.gov/ij/download.html, National Institutes of Health, USA). For each sample, at least 200 nanofibers from different SEM images were randomly selected and analyzed. Thermal gravimetric analysis (TGA) was performed on a TG209F1 system (NETZSCH Instruments Co., Ltd., Germany) at a heating rate of $10{ }^{\circ} \mathrm{C} \min ^{-1}$ under air atmosphere in a temperature range of $25-600{ }^{\circ} \mathrm{C}$. The porosity, mechanical 
properties, and the surface hydrophilicity of the nanofibers were measured according to protocols described in our previous work. ${ }^{25}$ Note that the bulk density of ATT is $2.18 \mathrm{~g} \mathrm{~cm}^{-3}$.

\section{Cell culture and cytocompatibility evaluation}

hMSCs were cultured in a humidified incubator with $5 \% \mathrm{CO}_{2}$ at $37^{\circ} \mathrm{C}$ using DMEM containing $10 \% \mathrm{FBS}, 100 \mathrm{U} \mathrm{mL}^{-1}$ penicillin, and $100 \mathrm{~g} \mathrm{~mL}^{-1}$ streptomycin. MTT assay and SEM observation were employed to evaluate the viability and morphology of the hMSCs cultured onto different nanofibers, respectively. Before cell seeding, glass cover slips, PLGA, and PLGA-ATT nanofibrous mats were placed in a 24-well tissue culture plate (TCP), fixed with stainless steel rings, sterilized with $75 \%$ alcohol for $2 \mathrm{~h}$, washed 3 times with PBS, and soaked in DMEM overnight. Then, hMSCs were seeded at a density of $2 \times 10^{4}$ cells per well for both the MTT assay and SEM observation. For comparison, TCPs and cover slips were used as controls. The protocols of MTT assay and SEM observation used in this study are similar to those described in our previous work. ${ }^{31}$

The viability of hMSCs cultured onto different nanofiber scaffolds at an extended period of time was also measured through the resazurin reduction assay according to protocols described in the literature. ${ }^{25,38}$ In brief, hMSCs were seeded at a density of $2 \times 10^{4}$ cells per well. At each predetermined time interval $(1,7,14$, and 21 days, respectively), medium in each well was replaced with a mixture of $100 \mu \mathrm{L}$ resazurin solution $\left(0.1 \mathrm{mg} \mathrm{mL} \mathrm{m}^{-1}\right.$ in PBS) and $900 \mu \mathrm{L}$ complete DMEM. After additional incubation of the cells for $4 \mathrm{~h}$, the fluorescence intensity was evaluated by a microplate reader (model Victor3 1420, PerkinElmer). Mean and standard deviation for the triplicate wells for each sample were reported.

\section{Hemocompatibility assays}

The hemocompatibility of the formed PLGA or PLGA-ATT nanofibers was examined by both hemolysis and anticoagulant assays. For hemolysis assay, human blood stabilized with heparin was centrifuged and washed with PBS for 5 times according to the procedures reported in the literature ${ }^{39}$ to completely remove serum and get human red blood cells (HRBCs). The HRBCs were diluted 10 times with PBS. Nanofibrous samples $(2 \mathrm{mg})$ were exposed to $1.0 \mathrm{~mL}$ HRBC suspension containing $0.2 \mathrm{~mL}$ diluted HRBC suspension and $0.8 \mathrm{~mL}$ PBS. At the same time, $0.2 \mathrm{~mL}$ of the diluted HRBC suspension was transferred to a Eppendorf tube pre-filled with $0.8 \mathrm{~mL}$ PBS (as negative control) or water (as positive control), respectively. The mixture was then incubated at $37^{\circ} \mathrm{C}$ for $2 \mathrm{~h}$, followed by centrifugation (10 $000 \mathrm{rpm}, 2 \mathrm{~min})$. The absorbance of the supernatant was recorded using a Lambda 25 UV-vis spectrophotometer (Perkin Elmer, Boston, MA). The hemolytic percentage (HP) was calculated by dividing the difference in absorbance $(540 \mathrm{~nm})$ between the sample and the negative control by the difference in absorbance $(540 \mathrm{~nm})$ between the positive and the negative control. ${ }^{40,41}$

The anticoagulant properties of the nanofiber samples were determined by a kinetic clotting time method as described in the literature. ${ }^{42-44}$ In brief, the nanofibrous mats were cut into small pieces in a dimension of $20 \times 20 \mathrm{~mm}^{2}$ in triplicate for each time point and put into individual wells of a 12-well TCP. Cover slips were used as control. Then, fresh human blood $(20 \mu \mathrm{L})$ was dropped into each well. A $\mathrm{CaCl}_{2}$ solution $\left(0.2 \mathrm{~mol} \mathrm{~L}^{-1}\right.$, $10 \mu \mathrm{L}$ ) was added to each blood drop and each sample was incubated at $37^{\circ} \mathrm{C}$ for different time periods $(5,10,20,40$, and $60 \mathrm{~min}$, respectively). This was followed by addition of $5 \mathrm{~mL}$ of water to each well and incubation at $37^{\circ} \mathrm{C}$ for $5 \mathrm{~min}$. A Lambda 25 UV-vis spectrophotometer was used to measure the absorbance of hemoglobin at $540 \mathrm{~nm}$.

\section{Osteogenic differentiation of hMSCs}

PLGA and PLGA-3\%ATT nanofibers were sterilized as mentioned above before cell seeding. TCP was set as control. hMSCs were seeded at a density of $2 \times 10^{4}$ cells per well with $1 \mathrm{~mL}$ of "growth medium" (DMEM containing $1 \%$ ascorbic acid (5 mg mL ${ }^{-1}$ in PBS) and $1 \%$ beta-GP solution ( $1 \mathrm{M}$ in PBS)) without DEX or $1 \mathrm{~mL}$ of "osteogenic medium" (growth medium containing $10^{-7} \mathrm{M}$ DEX) per well. All the cells were incubated at $37{ }^{\circ} \mathrm{C}$ in a humidified atmosphere with $5 \% \mathrm{CO}_{2}$ and the medium was replaced every 3 days.

\section{Protein content assay}

BCA Protein Assay Kit was used to quantify the protein content of each cell sample according to the manufacturer's instructions. After 14 and 21 days of culture onto different nanofibers, hMSCs cultured in a 24-well plate were rinsed 3 times with PBS. Reporter lysis buffer $(400 \mu \mathrm{L})$ was then added to each well and the cell lysis was carried out according to the manufacturer's instruction. Before analysis the cell lysates were stored at $-20^{\circ} \mathrm{C}$. To analyze the protein content, $20 \mu \mathrm{L}$ of each cell lysate or protein standards were transferred to a 96-well plate, followed by addition of $200 \mu \mathrm{L} \mathrm{BCA} \mathrm{working} \mathrm{reagent} \mathrm{to} \mathrm{each} \mathrm{well}$ and incubation of each sample at $37^{\circ} \mathrm{C}$ for $1 \mathrm{~h}$. The absorbance at $540 \mathrm{~nm}$ for each well was recorded using a microplate reader (MK3, Thermo, USA). The protein content was calculated from a standard calibration curve.

\section{Alkaline phosphatase activity}

Alkaline phosphatase (ALP) activity was determined by hydrolysis of $p$-nitrophenyl phosphate as the substrate. Briefly, $200 \mu \mathrm{L}$ of ALP substrate was mixed with $20 \mu \mathrm{L}$ of each cell lysate described above and the mixture was incubated for $1 \mathrm{~h}$ at $37^{\circ} \mathrm{C}$ in the dark. Then, $\mathrm{NaOH}(0.02 \mathrm{M}, 10 \mu \mathrm{L})$ was added to each well to stop the hydrolysis reaction. $220 \mu \mathrm{L}$ of ALP substrate mixed with $10 \mu \mathrm{L}$ of $0.02 \mathrm{M} \mathrm{NaOH}$ was used as a blank control. The absorbance was read at $405 \mathrm{~nm}$ using a microplate reader (MK3, Thermo, USA) and the ALP content was calculated from a standard calibration curve.

\section{Osteocalcin secretion assay}

Intact human osteocalcin EIA kit was used to measure the osteocalcin secretion of hMSCs on days 14 and 21. One day before the measurement, the medium was replaced with fresh growth medium or osteogenic medium without FBS. The next 
day, the medium was transferred to a clean Eppendorf tube, and the osteocalcin content was monitored using the intact human osteocalcin EIA kit according to the instructions of the manufacturer.

\section{Calcium content assay}

QuantiChrom Calcium Assay Kit was used to measure the calcium content according to the manufacturer's instructions. Briefly, $5 \mu \mathrm{L}$ of diluted standards or cell lysate were transferred to a 96-well plate. Followed by addition of $200 \mu \mathrm{L}$ working reagent and incubation for $3 \mathrm{~min}$ at room temperature, the optical density at $595 \mathrm{~nm}$ in each well was recorded using a microplate reader (MK3, Thermo, USA). The calcium content was calculated from a standard calibration curve.

\section{von Kossa staining of calcium deposition}

Before von Kossa staining, all the cells were first fixed with 3.7\% formaldehyde in PBS for $2 \mathrm{~h}$ at $4{ }^{\circ} \mathrm{C}$ and then rinsed with water for 3 times to remove all traces of formaldehyde. The fixed cells were treated with $2.5 \%$ silver nitrate solution under the exposure to ultra-violet light for $60 \mathrm{~min}$. The cells were then rinsed with water, treated with $5 \%$ sodium thiosulfate solution for 3 min, followed by rinsing with copious water. Finally, the cell samples were taken out from the culture plates and photographed using a digital camera (Samsung EK-GC110).

\section{Statistical analysis}

One-way ANOVA statistical method was used to evaluate the significance of the experimental data. A value of 0.05 was selected as the significance level, and the data were indicated with (*) for $p<0.05,(* *)$ for $p<0.01$, and $(* * *)$ for $p<0.001$, respectively.

\section{Results and discussion}

\section{Fabrication of electrospun ATT-doped PLGA nanofibers}

Similar to our previous work related to the incorporation of halloysite nanotubes, ${ }^{17,29,30}$ laponite nanodisks, ${ }^{25,31}$ nanohydroxyapatite ${ }^{26,27}$ within PLGA nanofibers, we were able to incorporate ATT nanoclay within PLGA nanofibers (Fig. 1). ATT displays an apparent rod shape with a mean diameter of $22.7 \pm 4.6 \mathrm{~nm}$ and quite a uniform size distribution (Fig. 1a).
The existence of ATT within ATT-doped PLGA hybrid nanofibers was visually confirmed by TEM (Fig. 1b), where individual ATT nanorods are coaxially aligned within the PLGA nanofibers. In contrast, PLGA nanofibers without ATT incorporation do not display rod-shaped particles within the nanofibers (Fig. 1c). TGA was used to further confirm the existence of the doped ATT within the hybrid nanofibers (Fig. S1, ESI $\dagger$ ). Apparently, PLGA polymer was burned out at a high temperature (above $400{ }^{\circ} \mathrm{C}$ ) and the ATT residue was left out. The mass residues of PLGA-1\%ATT, PLGA-2\%ATT, and PLGA-3\%ATT nanofibers at $600{ }^{\circ} \mathrm{C}$ were measured to be $0.98 \%, 1.87 \%$, and $3.17 \%$, respectively, in agreement with the initial ATT mass feeding percentages.

The morphology of the PLGA and ATT-doped PLGA nanofibers was observed by SEM (Fig. 2). In all cases, smooth and uniform nanofibers were able to be manufactured. It seems that the smooth and uniform fibrous morphology of PLGA nanofibers does not significantly change after the ATT incorporation. However, the diameter of PLGA nanofibers $(1308 \pm 296 \mathrm{~nm}$, Fig. 2a) decreased to $909 \pm 185 \mathrm{~nm}$ (Fig. 2b), $560 \pm 117 \mathrm{~nm}$ (Fig. 2c), and $483 \pm 133 \mathrm{~nm}$ (Fig. 2d), respectively after doping with $1 \%, 2 \%$, and $3 \%$ ATT. The fiber diameter decreased dramatically with the ATT incorporation level, which is presumably due to the change of the electrospinning solution properties after mixing with ATT. ${ }^{45}$ With the increase of the ATT incorporation level, the solution conductivity may increase due to the inherent ionic nature of ATT. ${ }^{\mathbf{4 6}}$ The decrease of the nanofiber diameter with the ATT incorporation level led to a variation in the porosity of the nanofibrous mat. As shown in Table S1 (ESI $\dagger$ ), after incorporation with 1-3\% ATT, the porosity of the hybrid nanofibrous mat decreases to $66-67 \%$ when compared with PLGA fibrous mat without ATT (72\%). This is likely attributed to the decreased fiber diameter after ATT incorporation.

The surface hydrophilicity of nanofibers is important for them to interact with cells. The influence of ATT incorporation on the surface hydrophilicity of PLGA nanofibers was then investigated (Fig. S2, ESI $\dagger$ ). It can be seen that the contact angle of PLGA nanofibers $\left(123.7 \pm 2.6^{\circ}\right)$ decreases to $120.2 \pm 3.7^{\circ}$, $116.8 \pm 1.5^{\circ}$, and $113.5 \pm 2.1^{\circ}$, respectively after incorporation with $1 \%, 2 \%$, and $3 \%$ ATT (Table S1 $\dagger$ ). This indicates that the hydrophilicity of the PLGA nanofibrous mat slightly increases with the doping amount of ATT, similar to the laponite-doped
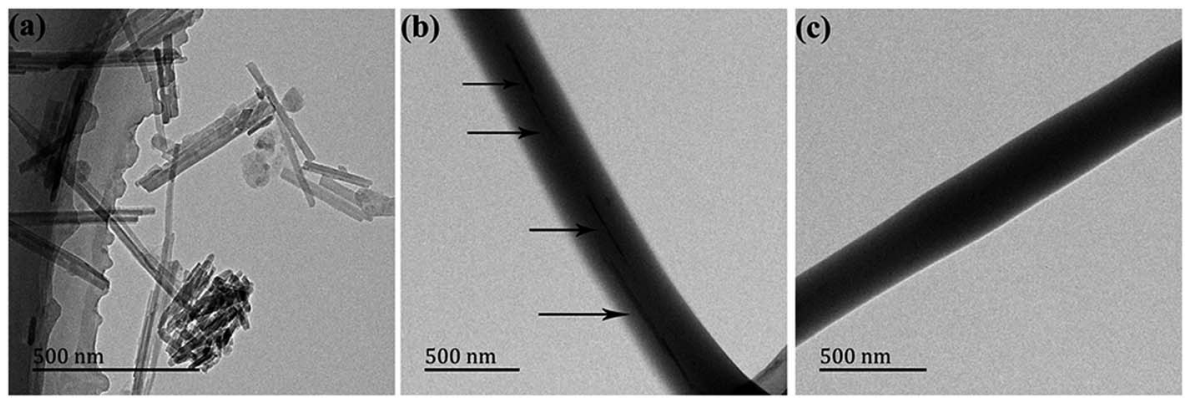

Fig. 1 TEM micrographs of ATT nanorods (a), PLGA-3\%ATT nanofibers (b), and PLGA nanofibers without ATT doping (c). In (b), the arrows indicate the doped individual ATT nanorods. 

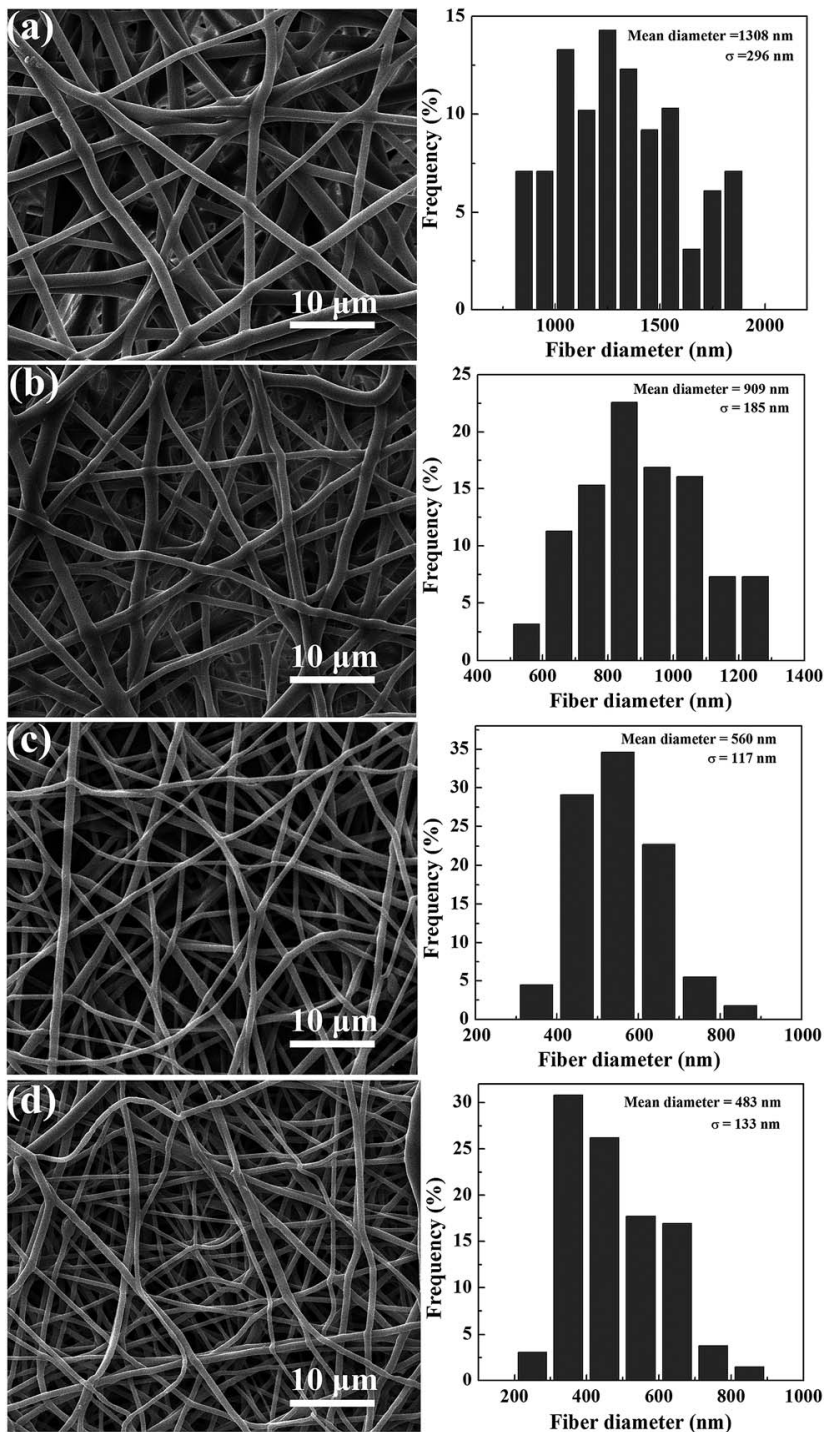

Fig. 2 SEM micrographs and diameter distribution histograms of PLGA (a), PLGA-1\%ATT (b), PLGA-2\%ATT (c), and PLGA-3\%ATT (d) nanofibers.

PLGA nanofibers reported in our previous work. ${ }^{25}$ Since the porosity of ATT-doped PLGA nanofibers remains approximately similar regardless of the ATT doping level (Table $\mathrm{S} 1 \dagger$ ), the increased hydrophilicity of the PLGA mats with the ATT incorporation level should be ascribed to the hydrophilic nature of the ATT. The increased hydrophilicity of the PLGA nanofibers after ATT incorporation may be beneficial to promote the infiltration of polar nutrient substances for improved cell attachment and proliferation.

Besides the changes of the fiber diameter, mat porosity, and the surface hydrophilicity of the PLGA nanofibers after ATT incorporation, the impact of ATT incorporation on the mechanical properties of the PLGA nanofibers was also explored. The strain-stress curves of the PLGA nanofibrous mats with or without ATT incorporation are shown in Fig. S3 (ESI $\dagger$ ), and the mechanical parameters are given in Table 1. It is clear that the breaking strength and Young's modulus increase after the ATT incorporation, in agreement with the literature data. ${ }^{17}$ The improved breaking strength and Young's modulus is believed to be attributed to the load transfer from the PLGA matrix to the incorporated ATT nanorods. However, the failure strain decreased after the incorporation with ATT, which is likely due to the increased brittleness of the fibers with the amount of ATT incorporation.

\section{Cytocompatibility assay}

For tissue engineering applications of the ATT-doped PLGA nanofibers, it is essential to explore the impact of the ATT incorporation on the cytocompatibility of the PLGA nanofibers. As shown in Fig. 3, it can be seen that the attachment of hMSCs cultured onto TCP, cover slips, and all fiber samples does not show any statistically significant difference after $8 \mathrm{~h}$ culture $(p>0.05)$. However, at the time point of $72 \mathrm{~h}$, the absorbance values are much higher than those onto the corresponding materials at $8 \mathrm{~h}(p<0.05)$, reflecting the proliferation of cells. Also, cell proliferation onto PLGA and all PLGA-ATT fibrous materials is much higher than that onto TCP $(p<0.05)$. This suggests that the incorporation of ATT does not compromise the inherent cytocompatibility of PLGA nanofibers, in agreement with our previous work associated with laponite-doped PLGA nanofibers. ${ }^{25}$ It should be pointed out that the viability of cells cultured onto PLGA and PLGA-ATT hybrid nanofibers (regardless of the amount of the incorporated ATT) does not display significant differences $(p>0.5)$. This is likely due to the fact that the incorporated ATT does not significantly impact the surface properties of the PLGA nanofibers.

The cytocompatibility of ATT-doped PLGA nanofibers was further confirmed by SEM observation of the morphology of hMSCs cultured onto the nanofiber scaffold for $8 \mathrm{~h}$ and 3 days, respectively (Fig. 4). It can be seen that hMSCs could be better adhered and proliferated onto the PLGA and ATT-incorporated PLGA nanofibers, and the incorporation of ATT does not impact the cytocompatibility of PLGA nanofibers, corroborating the quantitative MTT assay results.

Resazurin reduction assay was also used to test the impact of ATT incorporation within the PLGA nanofibers on the viability of hMSCs at an extended time period according to the literature. ${ }^{25,38}$ Fig. S4 (ESI $\dagger$ ) shows the resazurin reduction assay data of hMSCs at different time points. Obviously, from day 1 to day 7 , the fluorescence intensity has about 8 folds of increases, suggesting that hMSCs are able to rapidly grow and reproduce on TCP and PLGAbased nanofibers. Then, from day 7 to day 21, no significant difference in the fluorescence intensity is observed, which is likely ascribed to the saturation of cell growth on the fibrous scaffolds and TCPs. It is also clear that the metabolic activity of hMSCs cultured onto PLGA and all ATT-doped PLGA nanofibers does not show any significant difference when compared to the TCP control, demonstrating that hMSCs are able to proliferate well on different nanofiber scaffolds and the ATT-incorporation does not compromise the metabolic activity of the hMSCs. It should be noted that the metabolic activity of the hMSCs investigated here is different from that reported in our previous work. ${ }^{25}$ This may be due to the fact that the hMSCs are from different human tissues.

\section{Hemocompatibility assays}

For the applications of the hybrid nanofibers in therapeutic artificial tissue/organ substitutes in tissue engineering, one 
Table 1 Tensile properties of electrospun PLGA and composite PLGA nanofibers doped with ATT

\begin{tabular}{llcr}
\hline Sample & Breaking strength (MPa) & Failure strain (\%) & Young's modulus (MPa) \\
\hline PLGA & $6.50 \pm 0.47$ & $151.0 \pm 18.9$ & $164.16 \pm 1.42$ \\
PLGA-1\%ATT & $6.63 \pm 0.86$ & $106.9 \pm 21.4$ & $202.97 \pm 10.7$ \\
PLGA-2\%ATT & $6.83 \pm 1.15$ & $82.3 \pm 16.6$ & $219.57 \pm 7.89$ \\
PLGA-3\%ATT & $8.29 \pm 1.22$ & $116.1 \pm 7.9$ & $208.92 \pm 15.4$
\end{tabular}

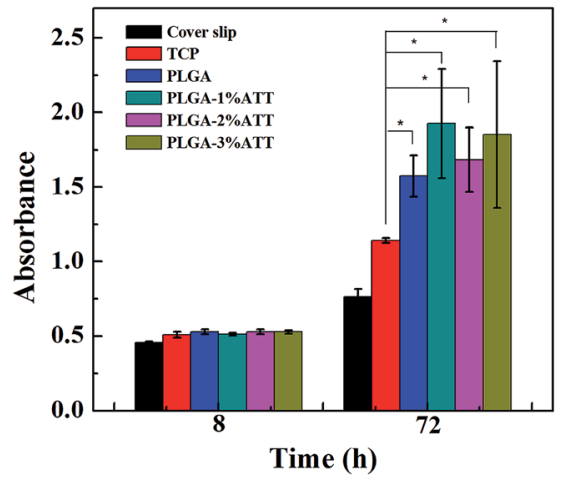

Fig. 3 MTT assay of the attachment and proliferation viability of hMSCs cultured onto cover slips, TCPs, and PLGA, PLGA-1\%ATT, PLGA-2\%ATT, and PLGA-3\%ATT nanofibrous mats, respectively (mean $\pm \mathrm{SD}, n=3$ ).

important concern is to check if the fibrous materials are hemocompatible. In this study, we investigated the hemocompatibility of PLGA and ATT-doped composite PLGA nanofibers with different doping levels via hemolysis assay and anticoagulant assay, respectively. As shown in the insets of Fig. 5, after immersion of all fiber samples to the HRBC suspension, no obvious hemolytic phenomenon can be observed except the positive control of water. The hemolytic effect of each material was further quantified by measuring the absorbance of the supernatant at $540 \mathrm{~nm}$ using UV-vis

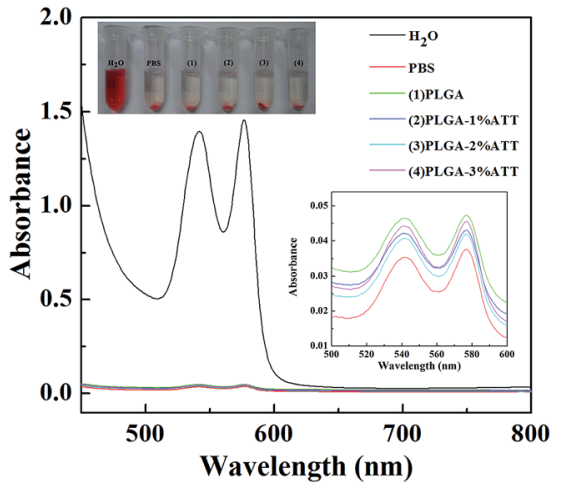

Fig. 5 UV-vis spectra of the HRBC suspensions treated with PLGA and ATT-doped PLGA nanofibers. The inset at the lower right corner shows an enlargement of the absorption features of the experimental groups. The inset at the upper left corner shows a photograph of HRBCs exposed to water, PBS, PLGA nanofibers (1), PLGA-1\%ATT nanofibers (2), PLGA-2\%ATT nanofibers (3), and PLGA-3\%ATT nanofibers (4) for $2 \mathrm{~h}$, followed by centrifugation.

spectroscopy, which is associated to hemoglobin from the lysed HRBCs. Apparently, significant difference $(p<0.001)$ in the absorbance at $540 \mathrm{~nm}$ between the experimental groups and the positive control group (the HRBCs exposed to water) can be seen. Furthermore, hemolysis percentages of the PLGA and all ATTdoped PLGA nanofibers (Table S2, ESI $\dagger$ ) were calculated to be less than the threshold value of $5 \%,{ }^{47}$ indicating that all the studied materials do not display appreciable hemolytic effect.
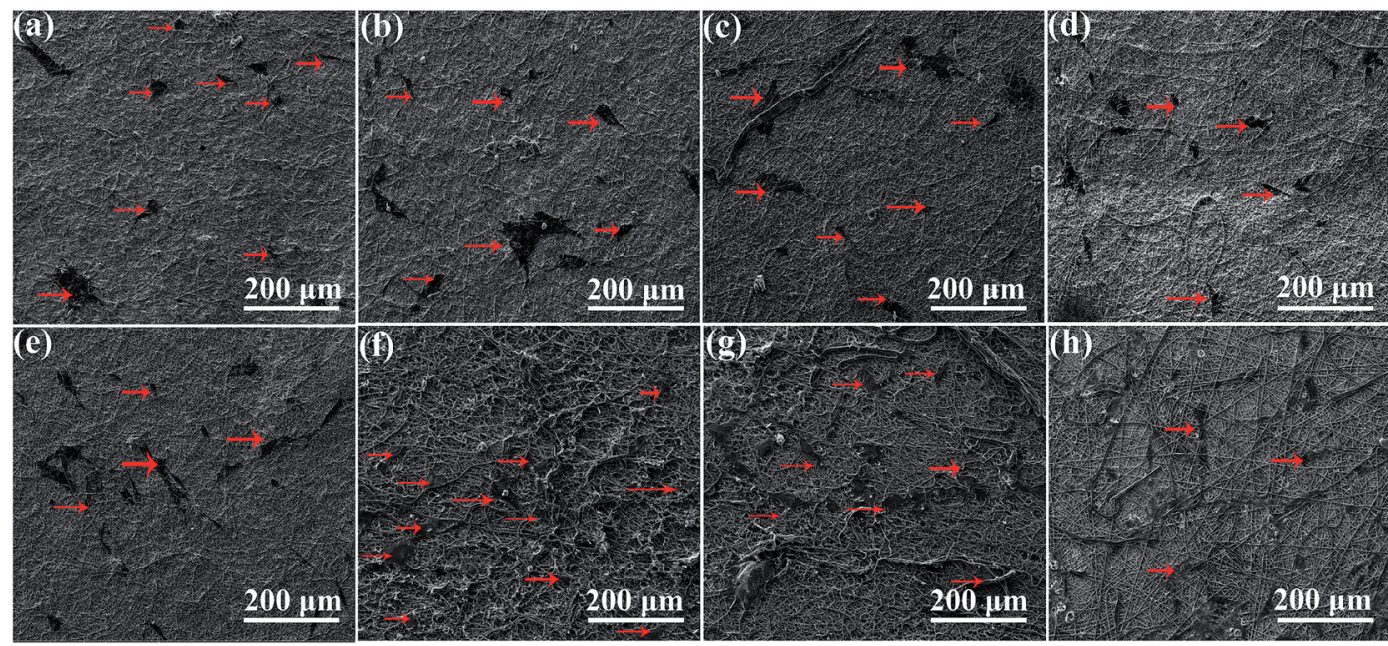

Fig. 4 SEM micrographs of hMSCs attached $(a-d)$ and proliferated (e-h) onto the PLGA (a and e), PLGA-1\%ATT (b and f), PLGA-2\%ATT (c and g), and PLGA-3\%ATT ( $d$ and $h$ ) nanofibrous mats after $8 \mathrm{~h}(\mathrm{a}-\mathrm{d})$ and 3 days $(\mathrm{e}-\mathrm{h})$, respectively. Red arrows indicate the hMSCs. 
The hemocompatibility of ATT-doped PLGA nanofibers was further confirmed by anticoagulant assay (Fig. S5, ESI $\dagger$ ). In general, a higher OD value representing a higher hemoglobin concentration suggests that the clotting behavior is less obvious. The OD values for the groups of PLGA and ATT-doped hybrid PLGA nanofibers are much higher than those for the control group of cover slips at each corresponding time point. In addition, there was no significant difference in the OD values $(p>0.05)$ between the PLGA nanofibers and the ATT-doped PLGA nanofibers with different ATT doping levels at each corresponding time point. This may be due to the fact that the ATT nanorods are well doped within the PLGA nanofibers, and the surface property of the hybrid ATT-doped PLGA nanofibers does not have apparent changes when compared with that of the PLGA nanofibers without doping. In contrast, under similar experimental conditions, cover slips show a significant clotting behavior. After incubation for $60 \mathrm{~min}$, the absorbance of hemoglobin is 0.18 , much lower than those of the fibrous samples (the lowest value of 0.44 at $60 \mathrm{~min}$ for PLGA-2\%ATT nanofibers). These results imply that all the PLGA nanofibrous mats with or without ATT doping display good anticoagulant property. Taken together with both the hemolysis and anticoagulation assay data, it is safe to conclude that the ATT-doped PLGA nanofibers possess good hemocompatibility.

\section{Osteogenic differentiation of stem cells}

The good cytocompatibility and hemocompatibility of ATTdoped PLGA nanofibers stimulated us to use them as a scaffold material for osteogenic differentiation of hMSCs, which is very important for tissue engineering applications. We selected PLGA-3\%ATT nanofibers as a model scaffold to investigate the role played by the doped ATT.

ALP activity of hMSCs cultured onto ATT-doped PLGA nanofibers was first explored to evaluate the osteogenic differentiation of hMSCs. ALP produced by active osteoblasts is involved in the early initiation of mineralization of newly formed bone tissue and has been identified to be an important early marker of osteogenesis. ${ }^{48}$ The ALP activity of hMSCs was measured on day 14 and day 21, and the results are normalized by the total protein content (Fig. 6). It is clear that, the ALP activity of hMSCs cultured onto TCP, PLGA, and PLGA-3\%ATT is low in both growth medium and osteogenic medium on day 14. On day 21, the hMSCs cultured onto different substrates in osteogenic medium supplemented with DEX have a significantly higher ALP activity than those cultured onto the corresponding substrates on day $14(p<0.05)$. Under similar experimental conditions, hMSCs cultured onto both PLGA and PLGA-3\%ATT nanofibers display a higher ALP activity than those cultured onto TCP. This suggests that the ECM mimicking property of PLGA nanofibrous scaffolds with or without ATT enables the osteogenic differentiation of hMSCs. In the presence of the inducing factor of DEX, PLGA and PLGA-3\%ATT nanofibers do not display any significant difference in terms of the ALP activity of the cultured hMSCs $(p>0.05)$.

Significantly different from the ALP activity of hMSCs cultured in osteogenic medium, the ALP activity of hMSCs

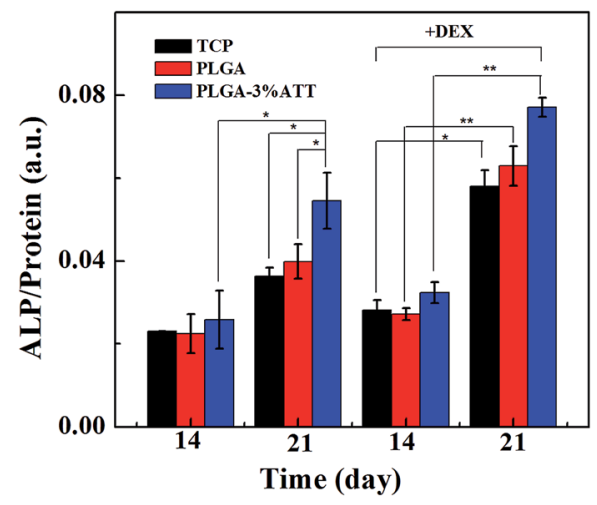

Fig. 6 ALP activity (normalized for the protein content, $\mathrm{n}$ mol of transformed substrate per unit of time and per mass of protein) of hMSCs cultured onto TCP, PLGA, and PLGA-3\%ATT nanofibers in growth medium (left part of the figure) and osteogenic medium (right part of the figure, labeled with "+DEX") at different culture times.

cultured in growth medium without DEX on day 21 is largely dependent on the type of the substrates. In contrast to the hMSCs cultured onto TCP and PLGA nanofibers that have increased ALP activity on day 21 when compared to those on day 14, the PLGA-3\%ATT nanofiber group shows a significantly higher ALP activity on day 21 than on day $14(p<0.05)$. More importantly, hMSCs cultured onto PLGA-3\%ATT nanofibers show a significantly higher ALP activity than those cultured onto TCP and PLGA nanofibers (day 21, $p<0.05$ ). This demonstrated that PLGA-3\%ATT nanofibers were able to regulate the osteogenic differentiation of hMSCs in growth medium without the inducing factor of DEX, similar to the laponite-doped PLGA nanofibers reported in our previous work. ${ }^{25}$

Osteocalcin is an important marker for late-stage osteogenic differentiation. The extracellular osteocalcin production after 14 and 21 days of culture was quantified to further confirm the osteogenic differentiation of hMSCs cultured onto different nanofibrous scaffolds (Fig. 7). In both growth and osteogenic medium, hMSCs cultured onto TCP, PLGA and PLGA-3\%ATT produced quite a low amount of osteocalcin on day 14. On day 21 , the osteocalcin production of hMSCs cultured in both growth medium and osteogenic medium was higher than that on day 14 , indicating the progression of osteogenic differentiation. In growth medium, the osteocalcin produced by hMSCs cultured onto PLGA-3\%ATT nanofibers was significantly higher than that onto PLGA nanofibers and TCP $(p<0.05)$. In contrast, no significant difference in the osteocalcin production $(p>0.05)$ between hMSCs cultured onto TCP and PLGA nanofibers was observed. This further confirmed the role played by the doped ATT that can regulate by itself the osteogenic differentiation of hMSCs in growth medium.

In osteogenic medium, from day 14 to day 21, the osteocalcin production from hMSCs cultured onto TCP, PLGA and PLGA-3\%ATT nanofibers showed a significant increase $(p<0.01)$, and the TCP control had much higher osteocalcin secretion than the same control in growth medium without DEX. This demonstrates that DEX is able to induce the 


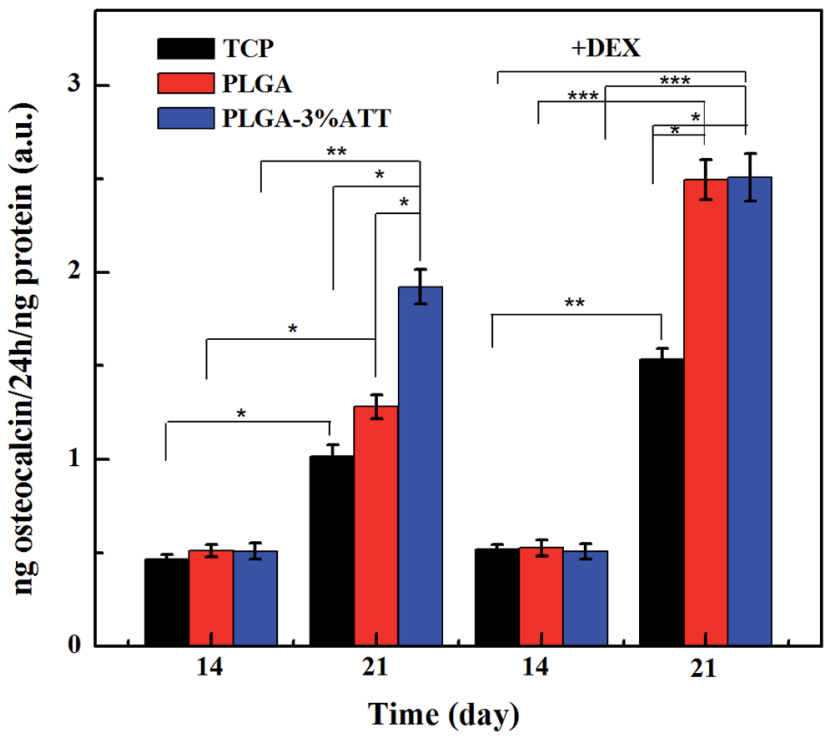

Fig. 7 Osteocalcin secretion of hMSCs cultured onto TCP, PLGA, and PLGA-3\%ATT nanofibers in growth medium (left part of the figure) and osteogenic medium (right part of the figure, labeled with "+DEX") at different culture times.

osteogenesis of hMSCs. Osteocalcin production from hMSCs cultured onto both PLGA and PLGA-3\%ATT nanofibers was higher than that onto the TCP control, in agreement with the ALP activity assay data. The osteocalcin assay demonstrates that ATT doping within PLGA nanofibers is able to regulate the osteogenic differentiation of hMSCs in the absence of the osteogenic inducing factor of DEX.

Calcium is another important marker for osteoblast differentiation of hMSCs. The calcium content of hMSCs cultured onto different substrates was analyzed on day 14 and day 21 to confirm the osteogenic differentiation of hMSCs (Fig. 8). It can be seen that the hMSCs cultured with osteogenic medium have relatively higher calcium content than that of the hMSCs cultured in growth medium without DEX under similar conditions (fiber substrate and time point). This tendency is more obvious on day 21, which is due to the inducing activity of the added DEX in the osteogenic medium. Importantly, hMSCs cultured onto PLGA-3\%ATT nanofibers in growth medium without DEX showed a significantly higher calcium content than those cultured onto ATT-free PLGA nanofibers and the TCP (day 21, $p<0.01$ ). These results further suggest that PLGA-3\% ATT nanofibers can induce osteogenic differentiation of hMSCs in growth medium without the inducing factor of DEX.

Finally, the presence of calcium phosphate crystals, which is a late-stage marker of osteogenic differentiation of hMSCs, ${ }^{49}$ was confirmed via von Kossa staining to qualitatively confirm the osteogenic differentiation of hMSCs (Fig. S6, ESI $\dagger$ ). Only the image of hMSCs cultured onto PLGA-3\%ATT nanofibers in growth medium is quite similar to those cultured onto ATT-free PLGA nanofibers and PLGA-3\%ATT nanofibers in osteogenic medium in terms of darkness. In contrast, hMSCs cultured onto ATT-free PLGA nanofibers in the growth medium do not display similar darkness after von Kossa staining. Therefore, the von-

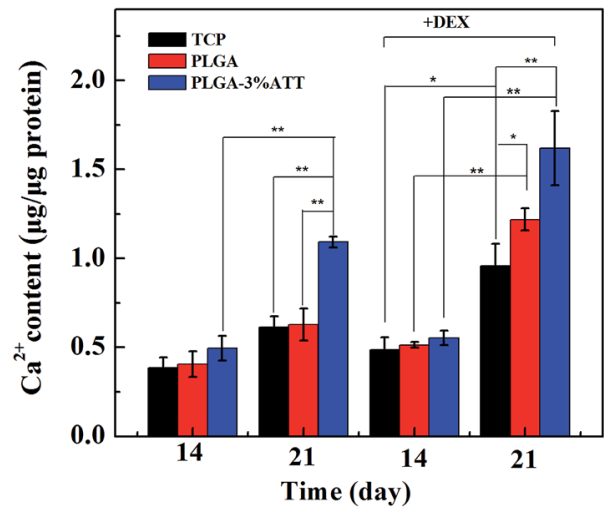

Fig. 8 Calcium content (normalized for the protein content) of hMSCs cultured onto TCP, PLGA, and PLGA-3\%ATT nanofibers in growth medium (left part of the figure) and osteogenic medium (right part of the figure, labeled with "+DEX") at different culture times.

Kossa staining assay qualitatively confirmed that PLGA-3\% ATT nanofibers were able to regulate osteogenic differentiation of hMSCs in the absence of the inducing factor of DEX. Likewise, due to the strong inducing effect of DEX, the inducing effect of the added ATT was not obvious from the results of von Kossa staining of hMSCs cultured onto the PLGA-3\%ATT nanofibers in osteogenic medium (with DEX).

\section{Conclusion}

In summary, electrospun ATT-incorporated PLGA nanofibers were fabricated and used as a scaffolding material for osteogenic differentiation of stem cells. Our results demonstrate that the ATT incorporation does not significantly change the uniform fibrous morphology and hemocompatibility of the PLGA nanofibers, instead leads to the reduction of the fiber diameter and porosity, the improved surface hydrophilicity, and the enhanced mechanical properties of the PLGA nanofibers. MTT cell viability assay and SEM observation of cell morphology reveal that the doped ATT within PLGA nanofibers is helpful to facilitate cell adhesion and proliferation of hMSCs when compared to pure PLGA nanofibers. Importantly, the ATT-doped PLGA nanofibers were able to be used as a scaffolding material for osteoblast differentiation of hMSCs in both growth and osteogenic medium. Our results show that the doping of ATT within the PLGA nanofibers enables osteoblast differentiation of hMSCs in growth medium without the inducing factor of DEX. The prepared smooth and uniform ATT-doped PLGA nanofibers may be used in different tissue engineering applications.

\section{Acknowledgements}

This research is financially supported by the High-Tech Research and Development Program of China (2012AA030309), the Program for Professor of Special Appointment (Eastern Scholar) at Shanghai Institutions of Higher Learning, and the FCT-Fundação para a Ciência e a Tecnologia (through the projects of PTDC/CTM-NAN/1748/2012, CQM 
pluriannual base funding of PEst-OE/QUI/UI0674/2011-2013). $\mathrm{X}$. S. gratefully acknowledges the University of Madeira and Santander bank for the Invited Chair in Nanotechnology.

\section{Notes and references}

1 D. Li and Y. N. Xia, Adv. Mater., 2004, 16, 1151-1170.

2 A. Greiner and J. H. Wendorff, Angew. Chem., Int. Ed., 2007, 46, 5670-5703.

3 H. Yoshimoto, Y. M. Shin, H. Terai and J. P. Vacanti, Biomaterials, 2003, 24, 2077-2082.

4 K. H. Lee, H. Y. Kim, M. S. Khil, Y. M. Ra and D. R. Lee, Polymer, 2003, 44, 1287-1294.

5 D. Li, Y. L. Wang and Y. N. Xia, Nano Lett., 2003, 3, 1167-1171. 6 Q. D. Tai, X. Z. Zhao and F. Yan, J. Mater. Chem., 2010, 20, 7366-7371.

7 J. J. Stankus, J. J. Guan, K. Fujimoto and W. R. Wagner, Biomaterials, 2006, 27, 735-744.

8 S. H. Choi, G. Ankonina, D. Y. Youn, S. G. Oh, J. M. Hong, A. Rothschild and I. D. Kim, ACS Nano, 2009, 3, 2623-2631.

9 W. J. Li, C. T. Laurencin, E. J. Caterson, R. S. Tuan and F. K. Ko, J. Biomed. Mater. Res., 2002, 60, 613-621.

10 W. Wang, H. M. Huang, Z. Y. Li, H. N. Zhang, Y. Wang, W. Zheng and C. Wang, J. Am. Ceram. Soc., 2008, 91, 38173819.

11 B. Ding, M. R. Wang, J. Y. Yu and G. Sun, Sensors, 2009, 9, 1609-1624.

12 C. Kim, Y. O. Choi, W. J. Lee and K. S. Yang, Electrochim. Acta, 2004, 50, 883-887.

13 Y. J. Yang, E. H. Liu, L. M. Li, Z. Z. Huang, H. J. Shen and X. X. Xiang, J. Alloys Compd., 2009, 487, 564-567.

14 K. Fujihara, A. Kumar, R. Jose, S. Ramakrishna and S. Uchida, Nanotechnology, 2007, 18, 365709.

15 I. D. Kim, J. M. Hong, B. H. Lee, D. Y. Kim, E. K. Jeon, D. K. Choi and D. J. Yang, Appl. Phys. Lett., 2007, 91, 163109.

16 M. S. Khil, D. I. Cha, H. Y. Kim, I. S. Kim and N. Bhattarai, J. Biomed. Mater. Res., 2003, 67, 675-679.

17 R. L. Qi, R. Guo, M. W. Shen, X. Y. Cao, L. Q. Zhang, J. J. Xu, J. Y. Yu and X. Y. Shi, J. Mater. Chem., 2010, 20, 10622-10629.

18 J. Zeng, X. Y. Xu, X. S. Chen, Q. Z. Liang, X. C. Bian, L. X. Yang and X. B. Jing, J. Controlled Release, 2003, 92, 227-231.

19 Y. K. Luu, K. Kim, B. S. Hsiao, B. Chu and M. Hadjiargyrou, J. Controlled Release, 2003, 89, 341-353.

20 E. R. Kenawy, G. L. Bowlin, K. Mansfield, J. Layman, D. G. Simpson, E. H. Sanders and G. E. Wnek, J. Controlled Release, 2002, 81, 57-64.

21 H. L. Jiang, Y. Q. Hu, Y. Li, P. C. Zhao, K. J. Zhu and W. L. Chen, J. Controlled Release, 2005, 108, 237-243.

22 X. L. Xu, L. X. Yang, X. Y. Xu, X. Wang, X. S. Chen, Q. Z. Liang, J. Zeng and X. B. Jing, J. Controlled Release, 2005, 108, 33-42.

23 H. X. Qi, P. Hu, J. Xu and A. J. Wang, Biomacromolecules, 2006, 7, 2327-2330.

24 S. Xiao, M. Shen, R. Guo, Q. Huang, S. Wang and X. Shi, J. Mater. Chem., 2010, 20, 5700-5708.
25 S. G. Wang, R. Castro, X. An, C. L. Song, Y. Luo, M. W. Shen, H. Tomás, M. F. Zhu and X. Y. Shi, J. Mater. Chem., 2012, 22, 23357-23367.

26 F. Y. Zheng, S. G. Wang, M. W. Shen, M. F. Zhu and X. Y. Shi, Polym. Chem., 2013, 4, 933-941.

27 F. Y. Zheng, S. G. Wang, S. H. Wen, M. W. Shen, M. F. Zhu and X. Y. Shi, Biomaterials, 2013, 34, 1402-1412.

28 S. Wang, Y. Zhao, M. Shen and X. Shi, Ther. Delivery, 2012, 3, 1155-1169.

29 R. Qi, R. Guo, F. Zheng, H. Liu, J. Yu and X. Shi, Colloids Surf., B, 2013, 110, 148-155.

30 R. Qi, X. Cao, M. Shen, R. Guo, J. Yu and X. Shi, J. Biomater. Sci., Polym. Ed., 2012, 23, 299-313.

31 S. Wang, F. Zheng, Y. Huang, Y. Fang, M. Shen, M. Zhu and X. Shi, ACS Appl. Mater. Interfaces, 2012, 4, 6393-6401.

32 C. Wang, S. Wang, K. Li, Y. Ju, J. Li, Y. Zhang, J. Li, X. Liu, X. Shi and Q. Zhao, PLoS One, 2014, 9, e99585.

33 Y. Huang, X. G. Jin, X. L. Zhang, H. L. Sun, J. W. Tu, T. T. Tang, J. Chang and K. R. Dai, Biomaterials, 2009, 30, 5041-5048.

34 Q. L. Yuan, W. B. Lu and Y. K. Pan, Polym. Degrad. Stab., 2010, 95, 1581-1587.

35 W. J. Wang, A. Li, J. P. Zhang and A. Q. Wang, Polym. Compos., 2007, 28, 397-404.

36 M. Tian, Y. Gao, Y. Liu, Y. L. Liao, R. W. Xu, N. E. Hedin and H. Fong, Polymer, 2007, 48, 2720-2728.

37 J. P. Zhang, Q. Wang and A. Q. Wang, Carbohydr. Polym., 2007, 68, 367-374.

38 S. Perrot, H. Dutertre-Catella, C. Martin, P. Rat and J. M. Warnet, Toxicol. Sci., 2003, 72, 122-129.

39 H. X. Wu, G. Liu, Y. M. Zhuang, D. M. Wu, H. Q. Zhang, H. Yang, H. Hu and S. P. Yang, Biomaterials, 2011, 32, 4867-4876.

40 Q. J. He, J. M. Zhang, J. L. Shi, Z. Y. Zhu, L. X. Zhang, W. B. Bu, L. M. Guo and Y. Chen, Biomaterials, 2010, 31, 1085-1092.

41 Y. S. Lin and C. L. Haynes, Chem. Mater., 2009, 21, 39793986.

42 Y. Imai and Y. Nose, J. Biomed. Mater. Res., 1972, 6, 165-172.

43 K. Y. Lee, W. S. Ha and W. H. Park, Biomaterials, 1995, 16, 1211-1216.

44 Z. X. Meng, W. Zheng, L. Li and Y. F. Zheng, Mater. Sci. Eng., C, 2010, 30, 1014-1021.

45 S. G. Wang, X. Y. Cao, M. W. Shen, R. Guo, I. Bányai and X. Y. Shi, Colloids Surf., B, 2012, 89, 254-264.

46 G. Tian, Y. Kang, B. Mu and A. Wang, Adsorpt. Sci. Technol., 2014, 32, 37-48.

47 Q. Z. Wang, X. G. Chen, Z. X. Li, S. Wang, C. S. Liu, X. H. Meng, C. G. Liu, Y. H. Lv and L. J. Yu, J. Mater. Sci.: Mater. Med., 2008, 19, 1371-1377.

48 C. Y. Wang, Y. R. Duan, B. Markovic, J. Barbara, C. R. Howlett, X. D. Zhang and H. Zreiqat, Biomaterials, 2004, 25, 2507-2514.

49 J.-S. Sun, W. H.-S. Chang, L.-T. Chen, Y.-C. Huang, L.-W. Juang and F.-H. Lin, Biomaterials, 2004, 25, 607-616. 